\title{
Response to Nicolaides' Comment
}

\author{
P. J. Aston \\ Department of Mathematics \\ University of Surrey \\ Guildford \\ Surrey GU2 7XH \\ $\mathrm{UK}$
}

December 22, 2015

In our paper [1], we suggested that non-exponential decay may occur for slowly decaying isotopes over long time periods, which is a regime that is difficult to access experimentally. In the discussion we noted that quantum mechanics does not predict exact exponential decay, and it is well known that theoretically there are deviations from exponential decay at very short times and at very long times [2], although both of these regimes are currently beyond the reach of experimental verification [3].

Nicolaides' Comment has considered aspects of this problem in more detail using quantum mechanics. In particular, he uses a formal solution to the time dependent Schrödinger equation which was derived in $[4,5]$ and plausible assumptions relevant to certain physical situations, together with experimental estimates of the two parameters $E_{r}$ and $\Gamma$, to argue that non-exponential decay for ${ }^{14} \mathrm{C}$ would only appear after hundreds of lifetimes, which would again be beyond experimental observation. In his earlier work, he also derived the expression for $P(t)$ given by equation (1) in his Comment which he claims holds for all $t$. Numerical evaluation of this function as shown in $[4,5]$ does indeed show an exponential region followed by non-exponential decay after many lifetimes for large $R\left(=E_{r} / \Gamma\right)$.

In response, we consider the form of non-exponential decay at short and long times as predicted by Nicolaides' model. In the earlier papers $[4,5]$, the complex function $G(t)$ was derived where the survival probability $P(t)=|G(t)|^{2}$. We note from equation (8.49) of [5] that as $t \rightarrow 0$,

$$
G(t) \simeq \ln \left[-(i / \hbar) z_{0} t\right]
$$

where $z_{0}=E_{r}-i \Gamma / 2$. This implies that as $t \rightarrow 0$,

$$
P(t)=|G(t)|^{2} \simeq \ln ^{2}[\Gamma t / \hbar]
$$

and so $P(t) \rightarrow \infty$ as $t \rightarrow 0$. Clearly this cannot be correct as $P(t)$ is a probability. It has been shown that for small $t$,

$$
P(t) \simeq 1-(\Delta H)^{2} t^{2}+O\left(t^{4}\right)
$$

which follows from an expansion of equation (48) in [2].

Moreover, for long times, Nicolaides work [4, 5] predicts non-exponential decay to be an inverse power law proportional to $t^{-2}$. Again, later work has shown that this long-time non-exponential decay is actually proportional to $t^{-3 / 2}[2]$. 
Thus, we note that the predictions from Nicolaides' model do not agree with the results of later work at very short or at very long times and so his prediction of exponential decay in between these extremes may not necessarily be correct either.

The predicted deviations from exponential decay at very short times and at very long times [2] are in the easiest regimes to study from a mathematical point of view as an asymptotic analysis can be performed based on either $t$ or $1 / t$ being small. However, our interest in the existence of non-exponential decay, with particular reference to the decay of ${ }^{14} \mathrm{C}$, is in the intermediate region between these extremes. The data available from dendrochronology that we analysed suggests the possibility of non-exponential decay of ${ }^{14} \mathrm{C}$ between one and two half lives [1]. This analysis was not based on any theory of radioactive decay but only on an analysis of experimental data.

To our knowledge, there is little theoretical work that has been done with regard to possible deviation from exponential decay between the extremes of very short and very long times and so there is a need for more careful calculations for individual physical systems in this intermediate time region. The experiment that we proposed in [1] would be one practical way to test our prediction of non-exponential decay for ${ }^{14} \mathrm{C}$, but theoretical work that addresses this intermediate regime would also be both valuable and interesting.

We conclude by quoting the beginning of the abstract of Peres [2]: "The decay of a nonstationary state usually starts as a quadratic function of time and ends as an inverse power law (possibly with oscillations). Between these two extremes, the familiar exponential decay law may be approximately valid." Who knows?!

\section{Acknowledgements}

I am grateful to Professor Ron Johnson for a useful discussion and some helpful comments on these issues.

\section{References}

[1] P.J. Aston. EPL 37, 52001, 2012.

[2] A. Peres. Ann. Phys. 129, 33, 1980.

[3] E.B. Norman, S.B. Gazes, S.G. Crane and D.A. Bennett. Phys. Rev. Letts 60, 2246, 1988.

[4] C.A. Nicolaides and D.R. Beck. Phys. Rev. Letts 38, 683, 1977.

[5] C.A. Nicolaides and D.R. Beck. Int. J. Quant. Chem. 14, 457, 1978. 